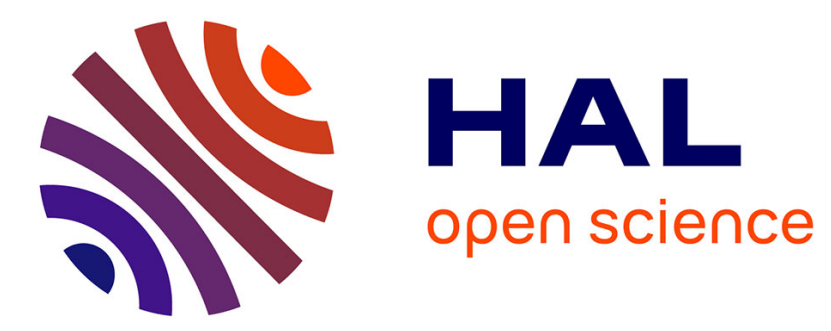

\title{
Sur un appareil pour la détermination des constantes magnétiques
}

\author{
Pierre Curie, C. Chéneveau
}

\section{To cite this version:}

Pierre Curie, C. Chéneveau. Sur un appareil pour la détermination des constantes magnétiques. J. Phys. Theor. Appl., 1903, 2 (1), pp.796-802. 10.1051/jphystap:019030020079600 . jpa-00240831

\section{HAL Id: jpa-00240831 https://hal.science/jpa-00240831}

Submitted on 1 Jan 1903

HAL is a multi-disciplinary open access archive for the deposit and dissemination of scientific research documents, whether they are published or not. The documents may come from teaching and research institutions in France or abroad, or from public or private research centers.
L'archive ouverte pluridisciplinaire HAL, est destinée au dépôt et à la diffusion de documents scientifiques de niveau recherche, publiés ou non, émanant des établissements d'enseignement et de recherche français ou étrangers, des laboratoires publics ou privés. 
SUR UN APPAREIL POUR LA DÉTERMINATION DES CONSTANTES MAGNÉTIQUES;

Par MM. P. CURIE et C. CHÉNEVEAU (1).

Cet appareil est destiné à mesurer les coefficients d'aimantation spécifique des corps faiblement magnétiques et diamagnétiques $\left({ }^{2}\right)$.

\section{I. - Principe et description.}

On mesure à l'aide d'une balance de torsion la force qui s'exerce sur un corps lorsqu'il est placé dans un champ magnétique non uniforme créé par un aimant permanent. La force est maximum pour une certaine position du corps par rapport à l'aimant : c'est cette position que l'on utilise dans les mesures. Le champ est créé par un aimant permanent NS, de forme annulaire, à pièces polaires biseautées et à entrefer assez étroit ( $f g .1$ et 2$)$. Le corps est placé dans un tube de verre $t$ fixé à l'une des extrémités d'une tige légère TT, en aluminium, suspendue en $\mathrm{O}$ à un fil de platine. A l'autre extrémité de la tige, se trouve placé un micromètre $m$ sur lequel est braqué un microscope M. Ce dispositif permet de suivre et de mesurer les déplacements de la balance de torsion.

Le tube $t$ contenant le corps est placé dans le plan de symétrie normal à la ligne des pôles, et il est attiré ou repoussé ( $f g$. 2) suivant la direction $a x$ normale à la ligne des pôles $\left({ }^{3}\right)$.

L'aimant est mobile; on peut le déplacer par translation (dans la direction indiquée par la flèche, $f g$. . 2). L'aimant étant d'abord éloigné du corps, si on l'approche de celui-ci, il se produit une attraction quand le corps est paramagnétique, une répulsion quand le corps est diamagnétique, et le mouvement du micromètre indique le sens de l'effet produit.

(1) Communication faite à la Société française de physique : Séance du 3 avril 1903.

(2) Nous rappellerons que le coefficient d'aimantation spécifique $\mathrm{K}$ est le rapport de l'intensité d'aimantation spécifique $\mathfrak{J}=\frac{\mathfrak{T}}{\mathrm{I}}$ ( $\mathcal{K}$, moment magnétique; $M$, masse) au champ magnétisant.

$\left.{ }^{3}\right)$ L'action du champ sur le corps est donnée par la lormule $f=\mathrm{KMH} y \frac{d \mathrm{H} y}{d x}$, dans laquelle $\mathrm{K}$ est le coefficient d'aimantation spécifique, $\mathrm{M}$ la masse, $\mathrm{H} y$ la valeur de l'intensité de champ dans la direction ay parallèle à la ligne des pôles, $\frac{d \mathrm{II} y}{d x}$ la dérivée du champ par rapport à une direction ax normale à celle du champ. 
Quel que soit l'effet initial observé, en approchant l'aimant d'unc façon continue, on constate que le déplacement du micromètre va d'abord en augmentant, passe par un maximum [fig. 2, position (1)

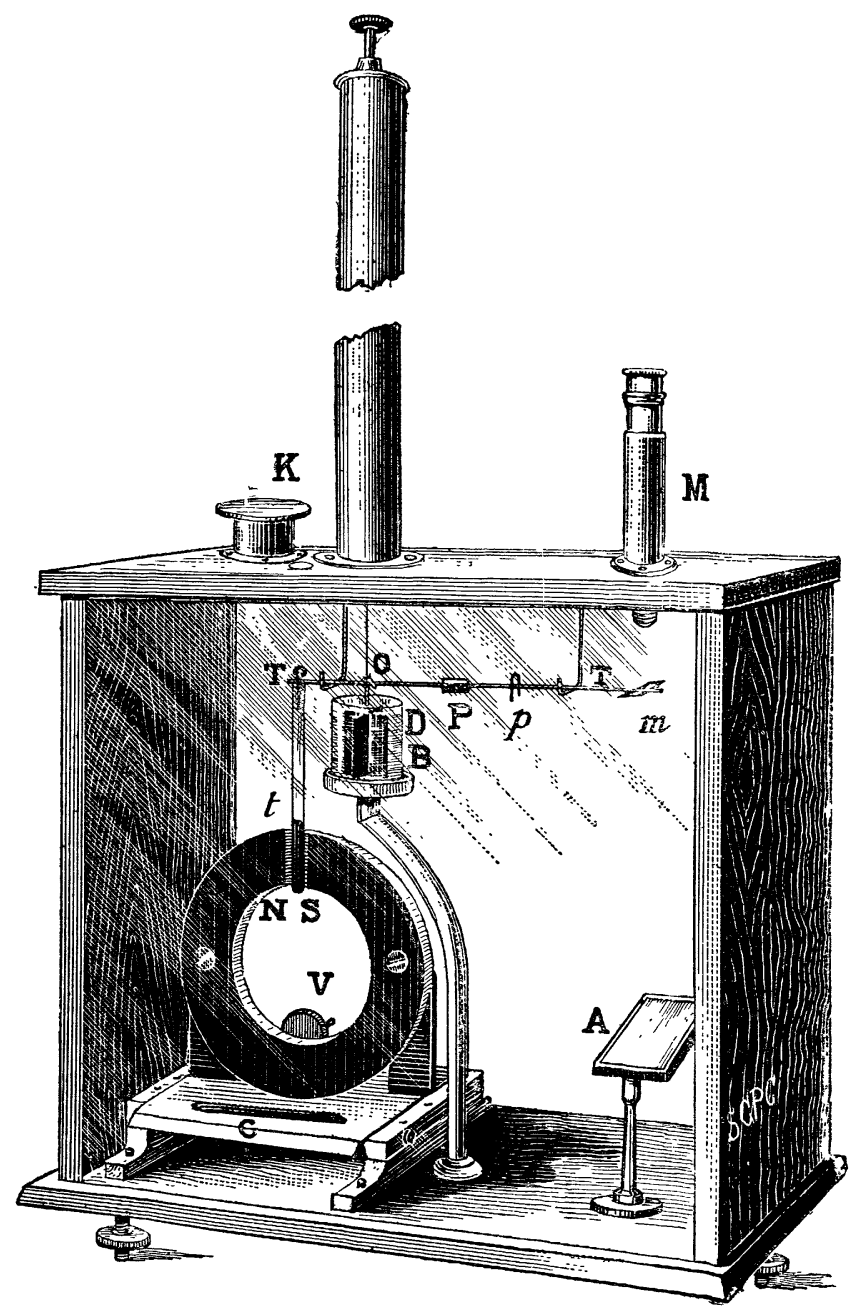

FIG. 1.

"de l'aimant et $t$ du tube], puis diminue pour s'annuler de nouveau quand le tube contenant le corps se trouve placé sur la ligne des pôles, entre les deux branches de l'aimant. La force s'annule en effet 
pour cette position symétrique. Si l'on continue à déplacer l'aimant dans le même sens, celui-ci passe de l'autre côté du corps et s'éloigne progressivement. La déviation indiquée par le micromètre change de sens, passe par un maximum [fg. 2, position (2) de l'aimant et $t^{\prime}$ du

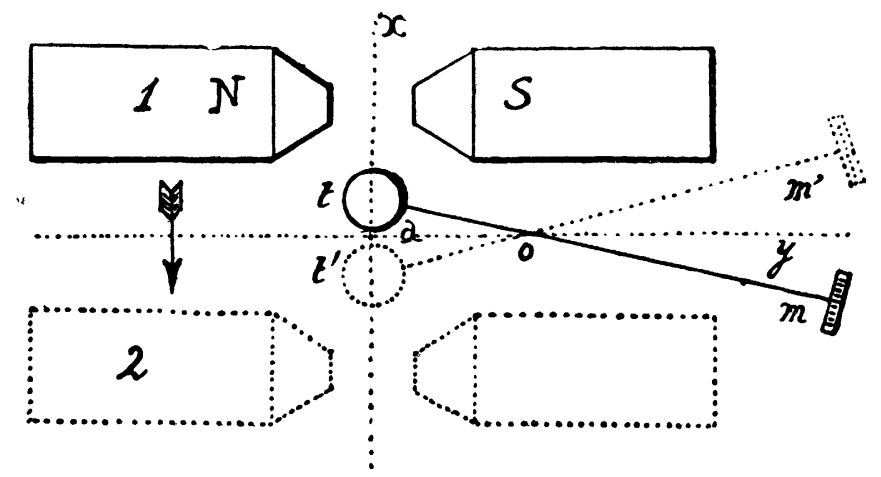

FIG. 2.

tube] et s'annule de nouveau quand l'aimant est suffisamment éloigné du corps. On note les divisions du micromètre qui coïncident avec le réticule du microscope pour les deux positions correspondant aux déviations maximum (positions $t$ et $t^{\prime}, f g .2$ ). Ces deux positions sont celles pour lesquelles la force passe par un maximum. La différence des lectures au micromètre est proportionnelle à la somme des deux valeurs maximum de la force, ces deux valeurs étant d'ailleurs égales entre elles, si l'appareil est symétrique.

Pour parfaire la description de l'appareil, nous indiquerons que le déplacement de l'aimant est obtenu en le rendant solidaire d'un chariot $\mathrm{C}$, guidé par deux glissières fixes, obéissant au mouvement direct ou rétrograde d'une vis $\mathrm{V}$ qui tourne dans un écrou fixé sous le chariot. L'une des glissières porte une graduation, le chariot mobile un trait de repère. L'équilibre de la balance de torsion se règle à l'aide d'un contrepoids cylindrique en laiton $P$ et d'un cavalier $p$ en aluminium. L'éclairage du micromètre se fait à l'aide du miroir A mobile dans plusieurs directions. L'amortissement est assuré par le frottement d'une palette $B$ en aluminium dans de l'huile de vaseline disposée dans le récipient $\mathrm{D}$. Un bouchon $\mathrm{K}$ permet d'enlever ou de placer le tube de verre sans ouvrir la cage de la balance. 
En ce qui concerne le tube de verre, il est suspendu par deux anneaux superposés, fixés à la tige 'TT. Le rebord qu'il porte s'appuie sur l'anneau supérieur, tandis que l'anneau inférieur le guide verticalement. L'appareil est réglé convenablement quand le trait de repère du chariot, coïncidant avec le zéro de la graduation de la glissière, le tube est symétriquement placé par rapport aux pièces polaires de l'aimant.

Le tube de verre de l'appareil étant soumis aux actions magnétiques aussi bien que la substance qu'il contient, il est nécessaire de faire une expérience avec le tube seul et de retrancher l'effet dû au tube de verre de l'effet total dû au tube rempli de substance. Il est avantageux de rendre cette correction faible en utilisant un verre à coefficient d'aimantation aussi petit que possible.

Le verre employé dans l'appareil est très légèrement diamagnétique à la température de $15^{\circ}$.

\section{II. - Mesures.}

$1^{\circ}$ Pour des mesures relatives, on prendra comme terme de comparaison un corps ou une solution dont le coefficient d'aimantation est connu.

Si $\Delta$ est la différence des lectures faites au micromètre pour une masse $m$ du corps, $\Delta^{\prime}$ le résultat d'une mesure faite avec une masse $m^{\prime}$ du corps de comparaison, $\Delta^{\prime \prime}$ la mesure lorsqu'on opère avec le tube de verre seul, $K$ et $\mathrm{K}^{\prime}$ les coefficients d'aimantation spécifique du corps à étudier et du corps de comparaison, le rapport de ces coefficients sera donné par la formule :

$$
\frac{\Delta-\Delta^{\prime \prime}}{\Delta^{\prime}-\Delta^{\prime \prime}}=\frac{\mathrm{K} m}{\mathrm{~K}^{\prime} m^{\prime}}
$$

Cette formule, vraie dans le cas où les trois corps sont paramagnétiques, sera généralisée d'après la convention suivante : On considérera les différences telles que $\Delta$ comme positives quand il s'agira d'une attraction (corps paramagnétique), et comme négatives s'il s'agit d'une répulsion (corps diamagnétique). Par exemple, la différence $\Delta$ dans le cas de l'eau est négative.

Toutefois la formule précédente n’est qu'approchée : la formule exacte doit en effet tenir compte du magnétisme de l'air. 
$2^{\circ}$ Correction due au magnélisme de l'air. Formule exacte. Soient $x^{\prime}, x^{\prime \prime}$, les susceptibilités en volume du corps de comparaison supposé magnétique, et de l'air. Soit $\mathrm{D}^{\prime}$ la densité du corps de comparaison et A une constante de l'appareil. En réalité, l'expression de la force quand on opère avec le corps de comparaison est :

$$
\mathbf{F}^{\prime}=\left(x^{\prime}-x^{\prime \prime}\right) \frac{m^{\prime}}{\mathrm{D}^{\prime}} \mathbf{A}
$$

or :

$$
\mathrm{K}^{\prime}=\frac{\varkappa^{\prime}}{\mathrm{D}^{\prime}}=\frac{\mathrm{F}^{\prime}}{m^{\prime} \mathrm{A}}+\frac{\varkappa^{\prime \prime}}{\mathrm{D}^{\prime}} .
$$

Lorsqu'on fait la mesure avec un corps paramagnétique de susceptibilité $x$ et de densité $\mathrm{D}$, la valeur réelle de la force est dans ce cas :

d'où :

$$
\mathrm{F}=\left(x-x^{\prime \prime}\right) \frac{m}{\mathrm{D}} \mathrm{A}
$$

Divisons les équations (4) et (3) membre à membre :

Posons :

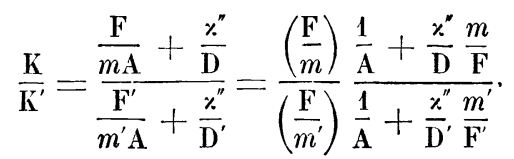

$$
r=\frac{\mathrm{F}}{\mathbf{F}^{\prime}} \cdot \frac{m^{\prime}}{m}=\frac{\Delta-\Delta^{\prime \prime}}{\Delta^{\prime}-\Delta^{\prime \prime}} \cdot \frac{m^{\prime}}{m} .
$$

C'est le rapport déterminé précédemment à l'aide de la formule (1):

$$
\frac{\mathrm{K}}{\mathbf{K}^{\prime}}=r \frac{1+\frac{\mathrm{A} m \varkappa^{\prime \prime}}{\mathrm{DF}}}{1+\frac{\mathrm{A} m^{\prime} \varkappa^{\prime \prime}}{\mathrm{D}^{\prime} \mathrm{F}^{\prime}}}=r\left[1+\mathrm{A} \varkappa^{\prime \prime}\left(\frac{m}{\mathrm{I} \mathrm{F}}--\frac{m^{\prime}}{\mathrm{D}^{\prime} \mathrm{F}}\right)\right] .
$$

D'où enfin, en remplaçant A par sa valeur tirée de l'équation (2) et le rapport $\frac{\mathrm{F}^{\prime}}{\mathrm{F}}$ par sa valeur tirée de l'équation $(\mathbf{5})$ :

$$
\frac{\mathrm{K}}{\mathbf{K}^{\prime}}=r\left[1+\frac{\varkappa^{\prime \prime}}{\varkappa^{\prime}-\varkappa^{\prime \prime}}\left(\frac{\mathrm{D}^{\prime}}{\mathrm{D} r}-1\right)\right]
$$


et comme $\mathrm{K}^{\prime}=\frac{x^{\prime}}{\mathrm{D}^{\prime}}$ :

$$
\frac{\mathrm{K}}{\mathrm{K}^{\prime}}=r\left[1+\frac{\varkappa^{\prime \prime}}{\mathrm{K}^{\prime}-\frac{\varkappa^{\prime \prime}}{\mathrm{D}^{\prime}}}\left(\frac{1}{\mathrm{D} r}-\frac{1}{\mathrm{D}^{\prime}}\right)\right] .
$$

On prendra pour coefficient d'aimantation en volume de l'air $x^{\prime \prime}$ la valeur $0,0322 \cdot 10^{-6}$ à $20^{\circ}\left(^{1}\right)$.

$3^{\circ}$ Le corps de comparaison pourra être en particulier un corps bien défini tel que l'eau. - Dans ce cas, la différence $\Delta$ sera négative. Si le verre est, comme nous l'avons indiqué, diamagnétique, $\Delta_{1}^{\prime \prime}$ sera également négatif. Ce sont les conditions que nous avons choisies pour l'emploi de l'appareil.

La formule générale (1), qui permet de connaître la valeur approchée de $\frac{\mathrm{K}}{\mathrm{K}^{\prime}}=r$, devient donc :

Pour un corps paramagnétique :

$$
\frac{\Delta_{1}+\Delta_{1}^{\prime \prime}}{-\Delta_{1}^{\prime}+\Delta_{1}^{\prime \prime}}=\frac{\mathrm{K}}{\mathrm{K}^{\prime}} \cdot \frac{m^{\prime}}{m}
$$

Pour un corps diamagnétique :

$$
\frac{-\Delta_{1}+\Delta_{1}^{\prime \prime}}{-\Delta_{1}^{\prime}+\Delta_{1}^{\prime \prime}}=\frac{\mathrm{K}}{\mathrm{K}^{\prime}} \cdot \frac{m^{\prime}}{m}
$$

formules dans lesquelles $\Delta_{1}, \Delta_{1}^{\prime}, \Delta^{\prime \prime}{ }_{1}$ sont les valeurs numériques particulières et absolues des différences observées dans l'expérience.

Comme d'ailleurs $\Delta_{1}^{\prime}>\Delta_{1}^{\prime \prime}$ en valeur absolue, il s'ensuivra que: pour un corps paramagnétique $r=\frac{\mathrm{K}}{\mathrm{K}^{\prime}}=-r_{1}$, et pour un corps diamagnétique $r=\frac{\mathrm{K}}{\mathrm{K}^{\prime}}=r_{1}, r_{1}$ étant une quantité positive.

Par suite également, la formule exacte générale (6) donnera :

a) Pour un corps faiblement magnétique :

$$
\frac{\mathrm{K}}{\mathrm{K}^{\prime}}=-r_{1}\left[1+\frac{0,0322}{0,79+0,03}\left(\frac{1}{r_{1} \mathrm{D}}+1\right)\right]
$$

puisque $x^{\prime \prime}$ pour l'air $=0,0322 \cdot 10^{-6} ;$

(1) P. Cunie, Annales de Chimie et de Physique, p. $344 ; 189$.

J. de Phys., $4^{\text {e }}$ série, t. II. (Novembre 1903.) 


\section{GURIE ET GHÉNEVAU. - GONSTANTES MAGNÉTIQUES}

Et K' pour l'eau peut être pris égal à $-0,79 \cdot 10^{-6}\left({ }^{1}\right)$;

Ou encore :

$$
\frac{\mathrm{K}}{\mathrm{K}^{\prime}}=-r_{1}\left[1+0,039\left(\frac{1}{r_{1} \mathrm{D}}+1\right)\right]
$$

b) Pour un corps diamagnétique :

$$
\frac{\mathrm{K}}{\mathrm{K}^{\prime}}=r_{1}\left[1-0,039\left(\frac{1}{r_{1} \mathrm{D}}-1\right)\right] .
$$

Pour l'application de ces diverses formules, il sera d'ailleurs facile et commode que les masses $m$ et $m^{\prime}$ se rapportent à un même volume : pour cela, on remplira le tube de verre jusqu'à un trait de repère.

$4^{\circ}$ Pour des mesures absolues, on pourra admettre que le coefficient d'aimantation spécifique de l'eau a la valeur précédemment indiquée, $-0,79 \cdot 10^{-6}$ à la température ordinaire, ce chiffre étant corrigé du magnétisme de l'air.

\section{III. - Expériences avec les sels de radium.}

Nous avons trouvé, avec cet appareil, que le chlorure de radium pur est paramagnétique. Son coefficient d'aimantation spécifique absolu, corrigé du magnétisme de l'air, comme il vient d'être indiqué, est : $1,03.10^{-6}$, en adoptant $-0,79 \cdot 10^{-6}$ pour le coefficient d'aimantation de l'eau qui nous a servi de corps de comparaison.

Le tableau suivant montre d'ailleurs qu'un produit contenant environ $1 / 6^{\text {e }}$ de chlorure de radium pour $5 / 6^{\text {e }}$ de chlorure de baryum est diamagnétique, son coefficient d'aimantation spécifique étant inférieur à celui du chlorure de baryum pur : ce qui confirme le fait précédemment énoncé :

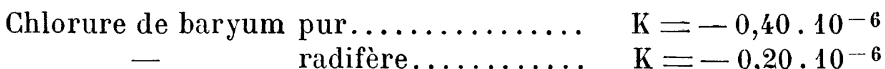

En 1899, M. St.-Meyer a annoncé que le carbonate de baryum radilère était paramagnétique $\left({ }^{2}\right)$. Cependant $M$. Meyer avait opéré avec un produit extrêmement peu riche, contenant peut-être $1 / 1.000^{\mathrm{e}}$ de radium, qui aurait dû être diamagnétique. Ce corps contenait peut-être une petite impureté ferrilère.

(1) Gcrie, loc. cit., p. 319.

(2) St -Meyer, Wied. Ann., t. LXVIII: 1899; - H. DU BoIs, Propriélés magnéiques de la matière pondérable, Congrès de Physique, p. $500 ; 1900$. 